\title{
Current status of $\varepsilon_{K}$ in lattice QCD
}

\section{Weonjong Lee*t}

Lattice Gauge Theory Research Center, CTP, and FPRD,

Department of Physics and Astronomy,

Seoul National University, Seoul 08826, South Korea

E-mail: wleedsnu.ac.kr

We present updated results of $\varepsilon_{K}$ evaluated directly from the standard model with lattice QCD inputs. Here, we use the lattice QCD inputs for $\hat{B}_{K},\left|V_{c b}\right|, \xi_{0}, \xi_{2},\left|V_{u s}\right|$, and $m_{c}\left(m_{c}\right)$. Recently, FLAG has updated $\hat{B}_{K}$. RBC-UKQCD has also updated $\xi_{0}$ and $\xi_{2}$. Exclusive $\left|V_{c b}\right|$ has been updated with new lattice data in the $\bar{B} \rightarrow D \ell \bar{v}$ decay mode, too. We find that the theoretical value of $\varepsilon_{K}$ with exclusive $\left|V_{c b}\right|$ (lattice QCD inputs) evaluated directly from the standard model is $3.2 \sigma$ lower than the experimental value, while that with inclusive $\left|V_{c b}\right|$ (heavy quark expansion) has no tension.

38th International Conference on High Energy Physics 3-10 August 2016

Chicago, USA

*Speaker.

†URL: http: //lgt.snu.ac.kr 


\section{Introduction}

We have been monitoring $\varepsilon_{K}$ since 2012, which is the indirect CP violation parameter in neutral kaons calculated directly from the standard model (SM) using lattice QCD inputs. The parameter $\varepsilon_{K}$ is very precisely measured in experiment. From the theoretical point of view, it comes from the FCNC loop effects of box diagrams in the SM, and so provide a direct probe of $\mathrm{CP}$ violation in the neutral kaon system. Hence, naturally it is sensitive to physics models beyond the standard model (BSM). In this paper, we present results of $\varepsilon_{K}$ evaluated directly from the SM with lattice QCD inputs. We also compare them with the experimental results. This paper is an update of our previous paper [1,2].

\section{Input parameters}

The master formula for $\varepsilon_{K}$ in the SM is

$$
\varepsilon_{K}=e^{i \theta} \sqrt{2} \sin \theta\left(C_{\varepsilon} X_{\mathrm{SD}} \hat{B}_{K}+\frac{\xi_{0}}{\sqrt{2}}+\xi_{\mathrm{LD}}\right)+\mathscr{O}\left(\omega \varepsilon^{\prime}\right)+\mathscr{O}\left(\xi_{0} \Gamma_{2} / \Gamma_{1}\right)
$$

Here, the short distance contribution proportional to $\hat{B}_{K}$ gives a contribution of about $105 \%$ of $\varepsilon_{K}$. The long distance effect, $\xi_{0}$ from the absorptive part gives about $-5 \%$ correction. The long distance effect, $\xi_{\mathrm{LD}}$ from the dispersive part gives about $\pm 1.6 \%$ correction. Details on remaining input parameters such as $C_{\varepsilon}, X_{\mathrm{SD}}, \xi_{0}$, and $\xi_{\mathrm{LD}}$ are given in Ref. [1]. We need 18 input parameters to determine $\varepsilon_{K}$ in the SM. Six of them can, in principle, be obtained from lattice QCD: $\hat{B}_{K}, V_{c b}$, $V_{u s}, \xi_{0}, \xi_{\mathrm{LD}}$, and $m_{c}\left(m_{c}\right)$. Here, we address recent progress on determining those input parameters.

\begin{tabular}{lll}
\hline \hline Decay mode & $\left|V_{u b}\right|$ & Ref. \\
\hline $\bar{B} \rightarrow \pi \ell \bar{v}$ & $3.72(16)$ & {$[3]$} \\
$\bar{B} \rightarrow \pi \ell \bar{v}$ & $3.61(32)$ & {$[4]$} \\
ex-combined & $3.70(14)$ & this paper \\
$\bar{B} \rightarrow X_{u} \ell \bar{v}$ & $4.45(16)(22)$ & {$[5]$} \\
\hline \hline
\end{tabular}

Table 1: Results for $\left|V_{u b}\right|$

\begin{tabular}{lll}
\hline \hline Decay mode & $\left|V_{c b}\right|$ & Ref. \\
\hline $\bar{B} \rightarrow D^{*} \ell \bar{v}$ & $39.04(49)(53)(19)$ & {$[6]$} \\
$\bar{B} \rightarrow D \ell \bar{v}$ & $40.7(10)(2)$ & {$[7]$} \\
ex-combined & $39.62(60)$ & this paper \\
$\bar{B} \rightarrow X_{c} \ell \bar{v}$ & $42.00(64)$ & {$[8]$} \\
\hline \hline
\end{tabular}

Table 2: Results for $\left|V_{c b}\right|$

Recent results for $\left|V_{u b}\right|$ and $\left|V_{c b}\right|$ are presented in Tables 1 and 2, respectively. Recently, DeTar has collected the lattice QCD results of FNAL/MILC [9] and HPQCD [10], and the experimental results of Babar [11] and Belle [12] for the $\bar{B} \rightarrow D \ell \bar{v}$ decay mode. He has made combined fit of all of them simultaneously to determine $\left|V_{c b}\right|$ [7]. The "ex-combined" result in Table 2 corresponds to a weighted average of the $V_{c b}$ results from the $\bar{B} \rightarrow D^{*} \ell \bar{v}$ and $\bar{B} \rightarrow D \ell \bar{v}$ decay channels. Similarly, the "ex-combined" result in Table 1 corresponds to a weighted average of the two $V_{u b}$ results from $\bar{B} \rightarrow \pi \ell \bar{v}$ decay. In Fig. 1 , we show all the results simultaneously. ${ }^{1}$ We find that the inclusive results show about $3 \sigma$ tension with those from exclusive $B$ meson decays respectively as well as from the LHCb results for $\left|V_{u b} / V_{c b}\right|$, which corresponds to the magenta band in Fig. 1.

We have two independent methods to determine $\xi_{0}$ in lattice QCD: the indirect and direct methods. In the indirect method, we determine $\xi_{0}$ from the experimental values of $\operatorname{Re}\left(\varepsilon^{\prime} / \varepsilon\right), \omega$,

\footnotetext{
${ }^{1}$ The plot is based on that by Andreas Kronfeld in Ref. [7].
} 


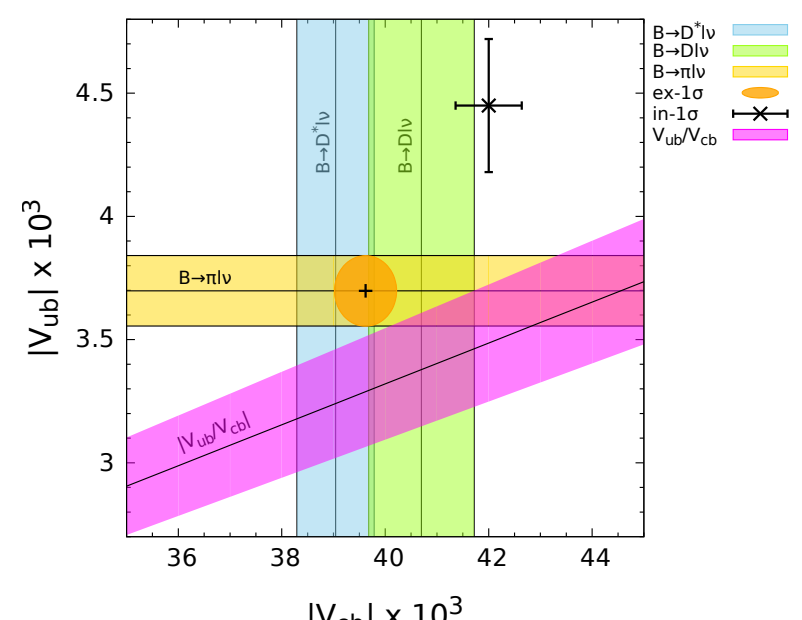

Figure 1: $\left|V_{u b}\right|$ versus $\left|V_{c b}\right|$. The sky-blue band represents $\left|V_{c b}\right|$ determined from the $\bar{B} \rightarrow$ $D^{*} \ell \bar{v}$ decay, and the yellow-green band $\left|V_{c b}\right|$ determined from the $\bar{B} \rightarrow D \ell \bar{v}$ decay. The yellow band represents $\left|V_{u b}\right|$ determined from the $\bar{B} \rightarrow \pi \ell \bar{v}$ decay, and the magenta band $\left|V_{u b} / V_{c b}\right|$ determined from the LHCb data of the $\Lambda_{b} \rightarrow \Lambda_{c} \ell \bar{v}$ and $\Lambda_{b} \rightarrow p \ell \bar{v}$ decays. The orange circle represents the combined results for exclusive $\left|V_{c b}\right|$ and $\left|V_{u b}\right|$ from the $B$ meson decays, and the black cross $\times$ the inclusive $\left|V_{c b}\right|$ and $\left|V_{u b}\right|$ (heavy quark expansion).

and $\varepsilon_{K}$ using the lattice QCD input $\xi_{2}$. The master formulas are

$$
\xi_{0}=\frac{\operatorname{Im} A_{0}}{\operatorname{Re} A_{0}}, \quad \xi_{2}=\frac{\operatorname{Im} A_{2}}{\operatorname{Re} A_{2}}, \quad \operatorname{Re}\left(\frac{\varepsilon^{\prime}}{\varepsilon}\right)=\frac{\omega}{\sqrt{2}\left|\varepsilon_{K}\right|}\left(\xi_{2}-\xi_{0}\right) .
$$

Recently, RBC-UKQCD reported updated results for $\xi_{2}$ [13]. The results for $\xi_{0}$ from the indirect method are presented in Table 3.

\begin{tabular}{llll}
\hline \hline Input & Method & Value & Ref. \\
\hline$\xi_{0}$ & indirect & $-1.63(19) \times 10^{-4}$ & {$[13]$} \\
$\xi_{0}$ & direct & $-0.57(49) \times 10^{-4}$ & {$[14]$} \\
\hline$\xi_{\mathrm{LD}}$ & - & $(0 \pm 1.6) \%$ & {$[15]$} \\
\hline \hline
\end{tabular}

Table 3: Long distance effects: $\xi_{0}$ and $\xi_{\mathrm{LD}}$.

\begin{tabular}{lll}
\hline \hline Collaboration & $\delta_{0}$ & Ref. \\
\hline RBC-UK-2016 & $23.8(49)(12)^{\circ}$ & {$[14]$} \\
KPY-2011 & $39.1(6)^{\circ}$ & {$[16]$} \\
CGL-2001 & $39.2(15)^{\circ}$ & {$[17,18]$} \\
\hline \hline
\end{tabular}

Table 4: $\pi-\pi$ scattering phase shift: $\delta_{0}$

Recently, RBC-UKQCD has reported new lattice QCD results for $\operatorname{Im} A_{0}$ [14]. Combining them with the experimental value of $\operatorname{Re} A_{0}$, we can determine $\xi_{0}$ directly from the lattice input $\operatorname{Im} A_{0} \mathrm{using}$ the master formula in Eq. (2.2). This is the direct method. In Ref. [14], RBC-UKQCD has also reported the $S$-wave $\pi-\pi$ scattering phase shift with isospin $I=0: \delta_{0}=23.8(49)(12)$. This value is $3.0 \sigma$ lower than the conventional value of $\delta_{0}$ in Refs. [16] (KPY-2011) and [17, 18] (CGL-2001). KPY-2011 used a singly subtracted Roy-like equation and CGL-2001 used a doubly subtracted Roy equation (CGL-2001) to do the interpolation around $\sqrt{s}=m_{K} \approx 500 \mathrm{MeV}$. The values for $\delta_{0}$ are summarized in Table 4. The KPY-2011 fits to the experimental data work well from the $\pi-\pi$ threshold $(\approx 280 \mathrm{MeV})$ to $\sqrt{s}=800 \mathrm{MeV}$. In addition, KPY-2011 is highly consistent with CGL2001 in the interpolating region around $\sqrt{s}=m_{K} \approx 500 \mathrm{MeV}$.

For $\delta_{0}(\mathrm{~S}$-wave, $\mathrm{I}=0)$, we plot the results of RBC-UKQCD together with those of KPY-2011 and CGL-2001 in Fig. 2. We find that there is essentially no difference between KPY-2011 and CGL-2001 in the region near $\sqrt{s}=m_{K} \approx 500 \mathrm{MeV}$. Here, we observe the $3.0 \sigma$ gap between RBC-UKQCD and KPY-2011. In contrast, for $\delta_{2}$ (S-wave, I=2), we observe no tension between RBC-UKQCD and KPY-2011, as one can see in Fig. 3.

Therefore, we conclude that the results of the indirect method are more reliable than those of the direct method for $\xi_{0}$, since the direct calculation of $\operatorname{Im} A_{0}$ by RBC-UKQCD might have unresolved issues. Hence, we use the indirect method to determine $\xi_{0}$ in this paper.

$\xi_{\mathrm{LD}}$ represents the long distance effect in the dispersive part. Its master formula in the continuum is given in Ref. [1]. A theoretical framework for calculating it on the lattice is well established 


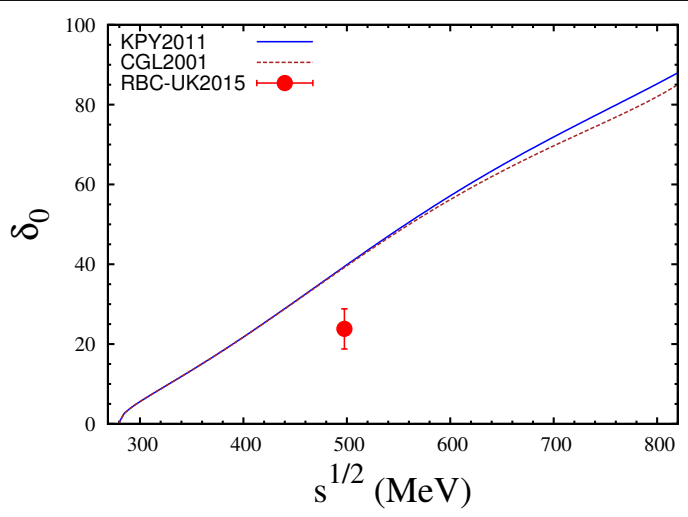

Figure 2: Comparison of $\delta_{0}$.

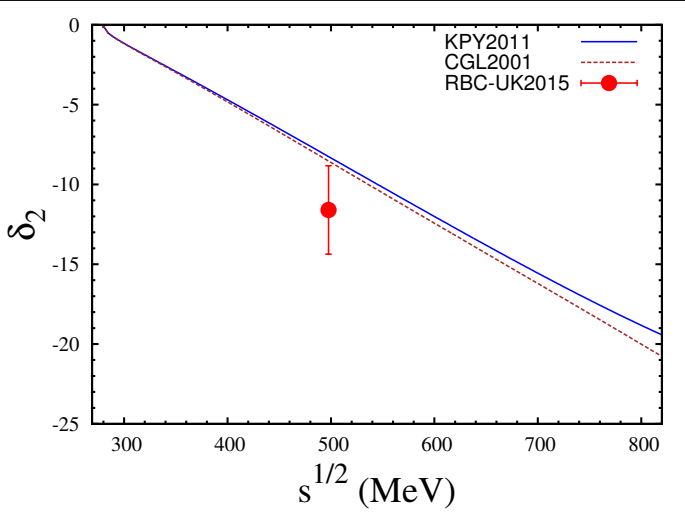

Figure 3: Comparison of $\delta_{2}$.

in Ref. [15]. An on-going efforts to calculate it on the lattice can be found in [19]. However, this attempt [20], at present, is in a exploratory stage yet. Hence, we use the rough estimate of $\xi_{\mathrm{LD}}$ given in Ref. [15].

Recent results for $\hat{B}_{K}$ in lattice QCD market with $N_{f}=2+1$ flavors are summarized in Table 5. Here, FLAG-2016 represents the global average of the results of BMW-2011 [21], Laiho-2011 [22], RBC-UK-2016 [23], and SWME-2016 [24]. For more details, refer to Ref. [25]. SWME2014 and RBC-UK-2016 represent the $\hat{B}_{K}$ results reported in Refs. [26] and [23], respectively. Here we use the FLAG-2016 result for $\hat{B}_{K}$.

\begin{tabular}{lll}
\hline \hline Collaboration & Value & Ref. \\
\hline FLAG-2016 & $0.7625(97)$ & {$[25]$} \\
SWME-2014 & $0.7379(47)(365)$ & {$[26]$} \\
RBC-UK-2016 & $0.7499(24)(150)$ & {$[23]$} \\
\hline
\end{tabular}

Table 5: $\hat{B}_{K}$

\begin{tabular}{llll}
\hline \hline & CKMfitter & UTfit & AOF \\
\hline$\lambda$ & $0.22548(68) /[27]$ & $0.22497(69) /[28]$ & $0.2253(8) /[29]$ \\
$\bar{\rho}$ & $0.145(13) /[27]$ & $0.153(13) /[28]$ & $0.139(29) /[30]$ \\
$\bar{\eta}$ & $0.343(12) /[27]$ & $0.343(11) /[28]$ & $0.337(16) /[30]$ \\
\hline
\end{tabular}

Table 6: Wolfenstein parameters

For the Wolfenstein parameters $\lambda, \bar{\rho}$, and $\bar{\eta}$, both CKMfitter and UTfit updated their results in Refs. [27, 28]. However, the angle-only-fit has not been updated since Lattice 2015. The global unitarity triangle (UT) fits of both CKMfitter and UTfit use $\varepsilon_{K}$ and $\left|V_{c b}\right|$ as input parameters to determine Wolfenstein parameters $\bar{\rho}$ and $\bar{\eta}$. Hence, using them to evaluate $\varepsilon_{K}$ leads to unwanted correlations through $\varepsilon_{K}$ and $\left|V_{c b}\right|$. In contrast, the angle-only-fit (AOF) results have no correlation with $\varepsilon_{K}$ and $\left|V_{c b}\right|$. Hence, we use the AOF results in this paper.

For the QCD corrections $\eta_{c c}, \eta_{c t}$, and $\eta_{t t}$, we use the same values as in Ref. [1]. They are collected in Table 7. In particular, we use the SWME value of $\eta_{c c}$ reported in Ref. [1] instead of that in Ref. [31]. This issue is well explained in Ref. [1]. One reason is that the size of the NNLO correction is already a conservative estimate for the truncation error of the NNNLO level in perturbation theory. Another reason is that the SWME result is consistent with that of Ref. [32].

In Table 8, we summarize remaining input parameters. They are the same as those in Ref. [1] except for the charm quark mass $m_{c}\left(m_{c}\right)$. For the charm quark mass, we use the HPQCD result reported in Ref. [35].

\section{Current status of $\varepsilon_{K}$}

Here, we present the results for $\varepsilon_{K}$ evaluated directly from the SM with the lattice QCD inputs 


\begin{tabular}{lll}
\hline \hline Input & Value & Ref. \\
\hline$\eta_{c c}$ & $1.72(27)$ & {$[1]$} \\
$\eta_{t t}$ & $0.5765(65)$ & {$[33]$} \\
$\eta_{c t}$ & $0.496(47)$ & {$[34]$} \\
\hline \hline
\end{tabular}

Table 7: QCD corrections.

\begin{tabular}{lll}
\hline \hline Input & Value & Ref. \\
\hline$G_{F}$ & $1.1663787(6) \times 10^{-5} \mathrm{GeV}^{-2}$ & {$[29]$} \\
$M_{W}$ & $80.385(15) \mathrm{GeV}$ & {$[29]$} \\
$m_{c}\left(m_{c}\right)$ & $1.2733(76) \mathrm{GeV}$ & {$[35]$} \\
$m_{t}\left(m_{t}\right)$ & $163.3(2.7) \mathrm{GeV}$ & {$[36]$} \\
$\theta$ & $43.52(5)^{\circ}$ & {$[29]$} \\
$m_{K^{0}}$ & $497.614(24) \mathrm{MeV}$ & {$[29]$} \\
$\Delta M_{K}$ & $3.484(6) \times 10^{-12} \mathrm{MeV}$ & {$[29]$} \\
$F_{K}$ & $156.2(7) \mathrm{MeV}$ & {$[29]$} \\
\hline \hline
\end{tabular}

Table 8: Other input parameters.

described in the previous section. Our preliminary results are, in units of $1.0 \times 10^{-3}$,

$$
\begin{array}{llrl}
\left|\varepsilon_{K}\right|=1.69 \pm 0.17 & & \text { for exclusive } V_{c b} \text { (lattice QCD) } \\
\left|\varepsilon_{K}\right|=2.10 \pm 0.21 & & \text { for inclusive } V_{c b} \text { (heavy quark expansion) } \\
\left|\varepsilon_{K}\right|=2.228 \pm 0.011 & & \text { (experimental value) }
\end{array}
$$

Here, exclusive $V_{c b}$ represents the theoretical evaluation of $\varepsilon_{K}$ with the FLAG-2016 $\hat{B}_{K}$, AOF for the Wolfenstein parameters, and exclusive $\left|V_{c b}\right|$ that corresponds to ex-combined in Table 2. We observe $3.2 \sigma$ tension in the exclusive $V_{c b}$ channel (lattice QCD), and no tension in the inclusive $V_{c b}$ channel (heavy quark expansion; QCD sum rules).

\section{Acknowledgments}

We thank R. Van de Water for helpful discussion on $V_{c b}$. The research of W. Lee is supported by the Creative Research Initiatives Program (No. 20160004939) of the NRF grant funded by the Korean government (MEST). W. Lee would like to acknowledge the support from the KISTI supercomputing center through the strategic support program for the supercomputing application research (No. KSC-2014-G3-003). The computations were carried out in part on the DAVID GPU clusters at Seoul National University.

\section{References}

[1] SWME Collaboration, J. A. Bailey, Y.-C. Jang, W. Lee, and S. Park Phys. Rev. D92 (2015), no. 3 034510, [1503.05388].

[2] J. A. Bailey, Y.-C. Jang, W. Lee, and S. Park PoS LATTICE2015 (2015) 348, [1511. 00969 ].

[3] Fermilab Lattice, MILC Collaboration, J. A. Bailey et al. Phys. Rev. D92 (2015), no. 1014024 , [1503.07839].

[4] J. M. Flynn, T. Izubuchi, T. Kawanai, C. Lehner, A. Soni, R. S. Van de Water, and O. Witzel Phys. Rev. D91 (2015), no. 7 074510, [1501.05373].

[5] Heavy Flavor Averaging Group (HFAG) Collaboration, Y. Amhis et al. 1412.7515.

[6] J. A. Bailey, A. Bazavov, C. Bernard, et al. Phys.Rev. D89 (2014) 114504, [1 403.0635$].$

[7] C. DeTar in Proceedings of the 27th International Symposium on Lepton Photon Interactions at High Energies, 2015. 1511.06884. 
[8] P. Gambino, K. J. Healey, and S. Turczyk 1606.06174.

[9] MILC Collaboration, J. A. Bailey et al. Phys. Rev. D92 (2015), no. 3 034506, [1503 . 07237 ].

[10] HPQCD Collaboration, H. Na, C. M. Bouchard, G. P. Lepage, C. Monahan, and J. Shigemitsu Phys. Rev. D92 (2015), no. 5 054510, [1505.03925]. [Erratum: Phys. Rev.D93,no.11,119906(2016)].

[11] BaBar Collaboration, B. Aubert et al. Phys. Rev. D79 (2009) 012002, [0 809 . 0828].

[12] R. Glattauer PoS EPS-HEP2015 (2015) 554.

[13] T. Blum et al. Phys. Rev. D91 (2015), no. 7 074502, [1 502 . 00263$].$

[14] RBC, UKQCD Collaboration, Z. Bai et al. Phys. Rev. Lett. 115 (2015), no. 21212001 , [1505.07863].

[15] N. Christ, T. Izubuchi, C. Sachrajda, A. Soni, and J. Yu Phys.Rev. D88 (2013), no. 1014508 , [1212.5931].

[16] R. Garcia-Martin, R. Kaminski, J. R. Pelaez, J. Ruiz de Elvira, and F. J. Yndurain Phys. Rev. D83 (2011) 074004, [1102.2183].

[17] G. Colangelo, J. Gasser, and H. Leutwyler Nucl. Phys. B603 (2001) 125-179, [hep-ph/0103088].

[18] https://indico.mitp.uni-mainz.de/event/48/contribution/5/material/ slides/0.pdf.

[19] N. Christ, T. Izubuchi, C. T. Sachrajda, A. Soni, and J. Yu PoS LATTICE2013 (2014) 397, [1402.2577].

[20] Z. Bai in Proceedings, 34th International Symposium on Lattice Field Theory (Lattice 2016): Southampton, UK, July 24-30, 2016, 2016. 1611.06601.

[21] S. Durr, Z. Fodor, C. Hoelbling, et al. Phys.Lett. B705 (2011) 477-481, [1106.3230].

[22] J. Laiho and R. S. Van de Water PoS LATTICE2011 (2011) 293, [1112 . 4861$].$

[23] RBC, UKQCD Collaboration, T. Blum et al. Phys. Rev. D93 (2016), no. 7 074505, [1411. 7017 ].

[24] SWME Collaboration, B. J. Choi et al., Kaon BSM B-parameters using improved staggered fermions from $N_{f}=2+1$ unquenched QCD, Phys. Rev. D93 (2016), no. 1 014511, [1509.00592].

[25] S. Aoki et al., Review of lattice results concerning low-energy particle physics, 1607.00299.

[26] T. Bae et al. Phys.Rev. D89 (2014) 074504, [1402 . 0048 ].

[27] J. Charles et al. Phys. Rev. D91 (2015), no. 7 073007, [1 501.05013$].$

[28] http://www.utfit.org/UTfit/ResultsSummer2016SM.

[29] K. Olive et al. Chin.Phys. C38 (2014) 090001.

[30] http://www.utfit.org/UTfit/ResultsSummer2014PostMoriondSM.

[31] J. Brod and M. Gorbahn Phys.Rev.Lett. 108 (2012) 121801, [1108. 2036].

[32] A. J. Buras and J. Girrbach Eur.Phys.J. C73 (2013) 2560, [1304 . 6835].

[33] A. J. Buras and D. Guadagnoli Phys.Rev. D78 (2008) 033005, [0 805 . 3887].

[34] J. Brod and M. Gorbahn Phys.Rev. D82 (2010) 094026, [1 007 . 068 4].

[35] B. Chakraborty, C. T. H. Davies, B. Galloway, P. Knecht, J. Koponen, G. C. Donald, R. J. Dowdall, G. P. Lepage, and C. McNeile Phys. Rev. D91 (2015), no. 5 054508, [1 408 . 4169].

[36] S. Alekhin, A. Djouadi, and S. Moch Phys.Lett. B716 (2012) 214-219, [1207 . 0980 ]. 